\title{
ONCHOCERCA SWEETAE CHEZ LE BUFFLE EN THAILANDE (1)
}

\author{
par O. Bain *, A.-G. Chabaud *, P. Wanantasame ud * c J. Nibhitabhata *** \\ - Laboratoire de Zoologie (Vers), associé au C.N.R.S., Muséum national d'Histoire naturelle. \\ 43, rue Cuvier, F 75231 Paris Cedex 05. \\ ** Bangkok Slaughter House, Soi Klueynam Thai Rd, Prakanong, Thailand. \\ to Thailand Institute of Scientific and Technological Research. \\ 196 Phahonyothin Rd., Bankhen, Bangkok, Thailand.
}

RESUME. L'Onchocerque intradermique chez Bubalus bubalis en Thaïlande est la même espèce que celle décrite sous le nom d'O. sweetce dans les nodules intradermiques de $B$. buba. lis en Australie.

Cette identification suggère qu'il n'y a pas eu de spéciation entre les parasites des Buffles d'Australie et d'Asie.

Le taxon O. indica Sweet, 1915, comprend deux espèces distinctes: l'une dans les muscles périmusculaires de Bos indicus est probablement $O$. gibsoni (et donc, l'espèce $O$. indica, basée essentiellement sur la description de ce matériel, doit tomber en synonymie); l'autre dans les nodules intradermiques de $B$. bubalis ne diffère pas d'O. sweetae. Pour admettre la distinction entre $O$. cebei, un parasite chez le même hôte d'Hanoï, et $O$. sweetae, il faudrait démontrer que la présence ou l'absence des papilles nettement précloacales est un caractère valide pour séparer ces espèces.

\section{Onchocerca sweetae occurring in the buffalo in Thailand.}

SUMMARY. The intradermal Onchocerca from Bubalus bubalis of Thailand is the same as that which was described as $\mathrm{O}$. sweetae from intradermal nodules in $\mathrm{B}$. bubalis from Australia.

This identification suggests there has been no speciation between the parasites of Australian and Asiatic Buffaloes.

The taxon O. indica Sweet, 1915 consists of two distinct species; one in perimuscular nodules of Bos indicus is most probably $\mathrm{O}$. gibsoni (and thus, the species $\mathrm{O}$. indica, based essentially on the description of this material, should fall in synonymy); the other in intradermal nodules of $\mathrm{B}$. bubalis does not differ from $\mathrm{O}$. sweetae.

To confirm the distinction between $\mathrm{O}$. cebei, a parasite in the same host from Hanoï, and $\mathrm{O}$. sweetae, it must be demonstrated that the presence or absence of clearly precloacal papillae is a valid character for separating these species.

(1) Ce travail a pu être effectué grâce à une subvention de l'Organisation Mondiale de la Santé. Accepté le 4 décembre 1979. 
Une dizaine de Bubalus bubalis sont examinés aux abattoirs de Bangkok, le 15 août 1979, pour rechercher des nodules à Onchocerques.

Aucun n'est trouvé au niveau des muscles thoraciques. Par contre, plusieurs nodules, de 0,5 à $2 \mathrm{~cm}$ de diamètre, sont récoltés dans l'épaisseur du derme, dans la région ventrale du thorax. Trois animaux sont infestés; deux ont un kyste, un en a une dizaine.

Parmi ces nodules, 3 sont plus ou moins calcifiés; les autres permettent de récolter, par dissection à frais ou digestion pepsique après fixation à l'alcool $70^{\circ}, 9 \uparrow$ et $8 \sigma^{\circ}$; chaque kyste contient une $q$ seule, $1 q$ et $1 \delta$, ou $1 q$ et $2 \delta$ (un cas); le plus petit kyste contient une mince $q$ immature et un jeune $\delta$ très court et grêle.

\section{Description du matériel de Bangkok.}

Les données morphologiques sont indiquées sur la figure 1.

Cuticule de la $q$ avec côtes ondulées et 4 stries par intercôte, rarement 3 ; musculature réduite et cordes latérales très développées. Queue conique.

Disposition des papilles caudales du $\delta$ peu variable; en particulier, chez tous les $\delta$, absence de papilles nettement précloacales et présence d'une petite paire de papilles submédianes, en arrière du cloaque.

Extrémité distale du spicule gauche conique ou de forme plus irrégulière, comme sur la figure $1 T$.

Microfilaire faiblement atténuée dans la région céphalique, à crochet très petit.

\section{Dimensions :}

- $q$ mûres: longueur du corps environ $120 \mathrm{~cm}$; corps large de 360 à $410 \mu \mathrm{m}$; œsophage long de 1350 à $1675 \mu \mathrm{m}$; vulve à $665-800 \mu \mathrm{m}$ de la tête; côtes espacées de $60-110 \mu \mathrm{m}$ et stries espacées de 15 à $40 \mu \mathrm{m}$; queue longue de 150 à $240 \mu \mathrm{m}$.

- $\varsubsetneqq$ immature : corps large de $180 \mu \mathrm{m}$, queue longue de $125 \mu \mathrm{m}$, côtes espacées de $30 \mu \mathrm{m}$ et stries espacées de $8 \mu \mathrm{m}$.

- $\sigma^{\star}$ adultes : corps long de 6,5 à $10,1 \mathrm{~cm}$ et large de 160 à $300 \mu \mathrm{m}$; anneau nerveux à 200-240 $\mu \mathrm{m}$ de l'apex; œesophage long de 1010 à $1900 \mu \mathrm{m}$; queue longue de 97 à $152 \mu \mathrm{m}$; spicule gauche long de 280 à $415 \mu \mathrm{m}$, avec manche de 110 à $165 \mu \mathrm{m}$; spicule droit long de 100 à $115 \mu \mathrm{m}$.

$-\sigma$ jeune : corps long de $3 \mathrm{~cm}$ et large de $60 \mu \mathrm{m}$; resophage long de $1010 \mu \mathrm{m}$; queue longue de $75 \mu \mathrm{m}$, spicule gauche et spicule droit longs de $320 \mu \mathrm{m}$ et $100 \mu \mathrm{m}$.

- Microfilaires mûres extraites de la partie terminale de l'ovéjecteur ( $q$ fixées à l'alcool) : corps long de 215 à $245 \mu \mathrm{m}(240,225,242,245,225,218,215,235,225$ et $230 \mu \mathrm{m})$ et large de 4 à $5 \mu \mathrm{m}$.

Un snip fait au niveau de la ligne médiane thoracique d'un Buffle parasité permet de récolter quelques microfilaires; l'une, mesurée après immobilisation à la chaleur, est longue de $225 \mu \mathrm{m}$.

\section{Identification.}

Tout un ensemble de caractères sont communs à notre matériel et à $O$. sweetae Spratt et Moorhouse, 1971, décrit également dans le derme de Bubalus bubalis, mais en Australie. Ce sont, chez la $q$, la cuticule à côtes ondulées et à 4 stries par intercôte *, la forme conique

* Ce caractère a été vérifié sur des fragments de $q$ d’O. sweetae provenant d'Australie, très aimablement donnés par le $\mathrm{D}^{\mathrm{r}}$ Moorhouse. 


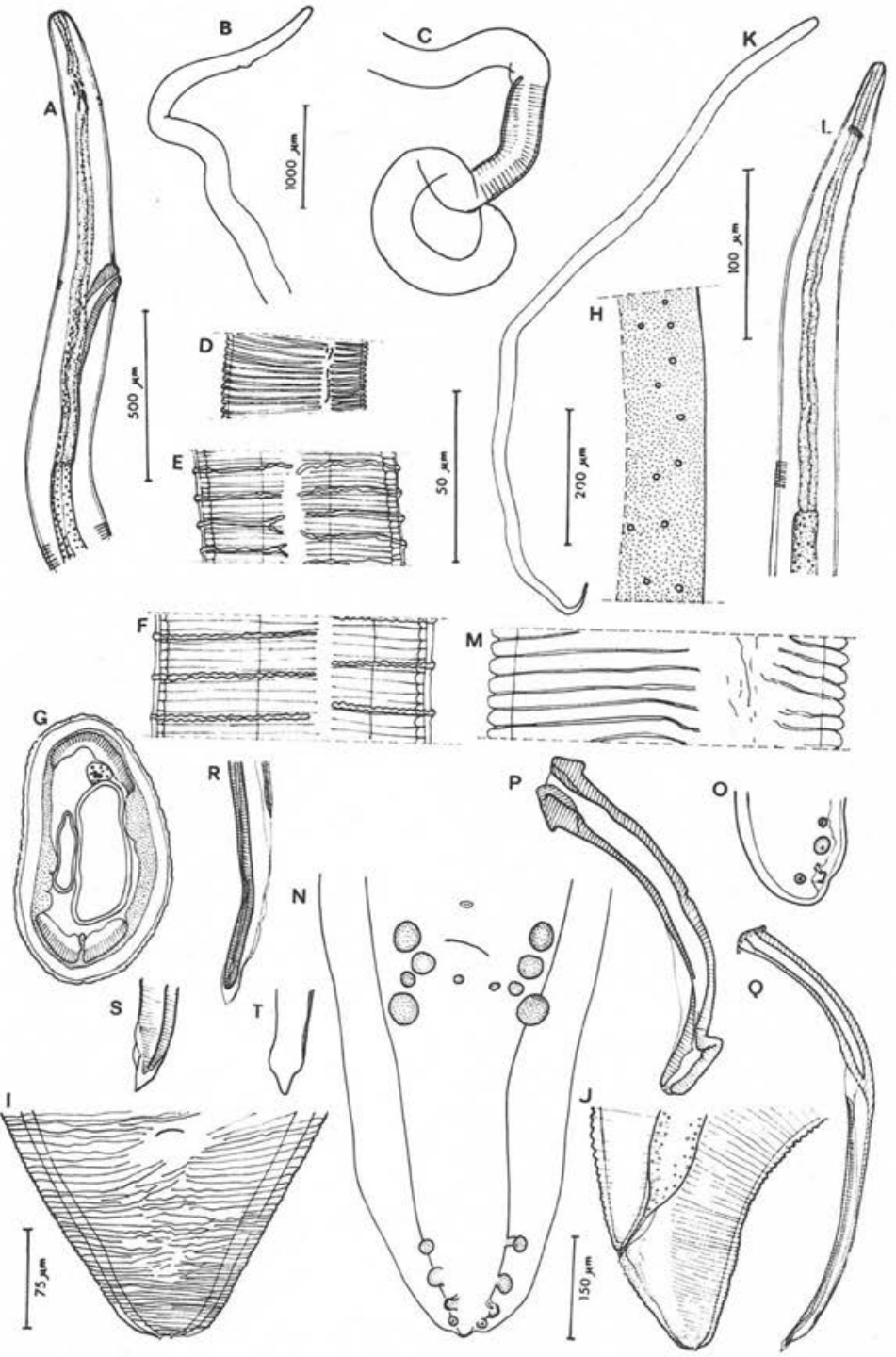

Fig. 1. Onchocerca sweetae

A à $\mathrm{K}, q$; $\mathrm{A}$ : région œsophagienne (stries cuticulaires représentées en 2 endroits); $\mathrm{B}$ et $\mathrm{C}$ : enroulement du corps dans les régions antérieure et postérieure du Ver; D et E : cuticule à $1800 \mu \mathrm{m}$ et $9000 \mu \mathrm{m}$ de la tête ; F : idem, à mi-corps, vue latérale ; G : même niveau, coupe transversale du Ver; $\mathrm{H}$ : demi-corde latérale avec files de noyaux ( $q$ d'Australie); $\mathrm{I}$ et $\mathrm{J}$ : queue, vues ventrale et latérale; $\mathrm{K}$ : microfilaire extraite de l'ovéjecteur; $\mathrm{L}$ à $\mathrm{T}, \widehat{\sigma}$; $\mathrm{L}$ : région œsophagienne, vue latérale; $\mathrm{M}$ : cuticule à mi-corps; $\mathrm{N}$ : queue, vue ventrale ; $\mathrm{O}$ : extrémité caudale, vue latérale; $\mathrm{P}$ : spicule droit, vue latérale gauche; $\mathrm{Q}$ : spicule gauche disséqué, vue latérale; $\mathrm{R}, \mathrm{S}$ et $\mathrm{T}$ : idem, détail de l'extrémité distale de 3 spicules gauches, en vues latérale, dorsale et ventrale.

(A et L : éch. $500 \mu \mathrm{m}$; B et C : éch. $1000 \mu \mathrm{m} ; \mathrm{D}, \mathrm{E}, \mathrm{F}, \mathrm{G}, \mathrm{J}: 150 \mu \mathrm{m}$; M : éch. $100 \mu \mathrm{m}$; $\mathrm{H}$ : éch. $200 \mu \mathrm{m}$; I : éch. $75 \mu \mathrm{m}$; K, N, O, P, Q, R, S, T : éch. $50 \mu \mathrm{m}$ ). 
de la queue ; chez le $\delta$, la disposition des papilles caudales (pas de papilles très antérieures au cloaque, 1 paire de petites papilles subventrales postcloacales), la taille souvent élevée des spicules.

Seules les dimensions des microfilaires diffèrent : 246-281 $\mu \mathrm{m}$ sur 5,5 $\mu \mathrm{m}$ selon Spratt et Moorhouse, 215-245 $\mu \mathrm{m}$ sur 4-5 $\mu \mathrm{m}$ pour le matériel de Bangkok. Mais nous pensons que ces différences résultent des techniques d'observation **: sur "thin smears " par Spratt et Moorhouse, ce qui favorise l'étirement et l'aplatissement des microfilaires; sur microfilaires extraites de l'ovéjecteur de $q$ fixées à l'alcool dans le cas de notre matériel.

Nous estimons donc qu'aucun caractère valable n'oppose le matériel de Bangkok à $O$. sweetae; nous concluons donc à cette identification.

\section{Onchocerques nodulaires des Bovidés domestiques en Asie et en Australie.}

4 noms différents se trouvent dans la littérature : O. gibsoni Cleland et Johnston, 1910, O. sweetae, O. indica Sweet, 1915 et O. cebei Galliard, 1937. Les 2 derniers taxa reposent sur des descriptions anciennes et engendrent, de ce fait, une certaine confusion.

Bien qu'il ne nous ait pas été possible de réétudier les matériels types de ces 2 espèces, il nous semble que les problèmes posés par celles-ci peuvent être en partie simplifiés.

a. O. cebei, chez Bubalus bubalis, dans le derme au Tonkin.

$O$. sweetae a été décrite comme très proche d'O. cebei, tant par l'hôte parasité que par la morphologie. Les caractères de la cuticule (cf. ci-dessus), confirment encore ces similitudes: dans les 2 cas, il y a 4 stries par intercôte.

La distinction entre les 2 espèces ne tient donc, comme l'ont écrit Spratt et Moorhouse, qu'à la disposition des papilles caudales : chez $O$. sweetae, il n'y a pas de papilles nettement antérieures au cloaque (les $\delta َ$ observés à Bangkok sont également de ce type), chez $O$. cebei, ces papilles paraissent être présentes (un dessin donné par Gailliard).

b. $O$. indica, aux Indes.

Le matériel étudié par Sweet aux Indes, sur lequel est basée l'espèce $O$. indica, comprend des spécimens extraits de nodules périmusculaires chez Bos indicus et des spécimens extraits de nodules dermiques chez Bubalus bubalis.

L'analyse du long travail rédigé par G. Sweet et les connaissances morphologiques établies récemment permettent de conclure que l'auteur a eu affaire, en réalité, à 2 espèces distinctes :

1. Nodules périmusculaires chez Bos indicus.

Sweet constate des différences nettes entre les dimensions de son matériel indien et celles d'O. gibsoni (données de Gilruth et Sweet, 1911). En fait, les dimensions d'O. gibsoni varient de façon importante et ces caractères différentiels se révèlent sans valeur, comme l'indiquent les nombres suivants (pour chaque caractère sont données successivement les mensurations d'indica, puis celles de gibsoni, de Bain et Beveridge, 1979): chez le ó: œsophage long de 847-1 $220 \mu \mathrm{m}$ et $1050-1120 \mu \mathrm{m}$; spicule gauche long de 207-276 $\mu \mathrm{m}$ et 180-260 $\mu \mathrm{m}$; spicule droit long de 82-94 $\mu \mathrm{m}$ et $80-90 \mu \mathrm{m}$; chez la $q$ : œesophage long de $1100-1440 \mu \mathrm{m}$ et $990-1130 \mu \mathrm{m}$.

Enfin, la longueur de la microfilaire $(120-160 \mu \mathrm{m})$ ne peut être prise en considération, puisqu'il s'agit de "larva just escaped from egg ».

** Les fragments $\subsetneq$ d'Australie ne renferment que des microfilaires immatures (longues de $180 \mu \mathrm{m})$, inutilisables pour une étude comparée. 
La longueur du spicule gauche $(207-276 \mu \mathrm{m})$ et le fait que les stries cuticulaires entre les côtes sont relativement peu visibles (elles ont été mises en évidence pour la première fois chez gibsoni par Johnston, en 1921) montrent qu'il s'agit de gibsoni et non de sweetae.

2. Nodules intradermiques chez Bubalus bubalis.

Aucune des mensurations ou des données morphologiques indiquées par Sweet pour le matériel indien provenant du Buffle ne s'éloigne de celles que nous observons en Thaïlande dans le matériel provenant du même hôte *.

La présence de 4 stries par intercôte est l'élément déterminant qui permet d'écarter le diagnostic d'O. gibsoni et de rattacher ce matériel à ce que nous désignons actuellement sous le nom d'O. sweetae.

En conclusion, les Onchocerques parasites intradermiques de Bubalus bubalis en Thaïlande correspondent à celles qui se trouvent chez le même hôte et dans la même localisation en Australie, et nous les désignons sous le nom d'O. sweetae.

Cette identification rend peu plausible l'existence de spéciations entre les parasites des Buffles australiens et des Buffles asiatiques.

Le taxon $O$. indica Sweet, 1915 recouvre, en réalité, 2 espèces distinctes: l'une, dans les kystes périmusculaires de Bos indicus, est très vraisemblablement $O$. gibsoni (et l'espèce indica, fondée essentiellement sur la description de ce matériel, tomberait ainsi en synonymie) ; l'autre, dans les kystes dermiques de Bubalus bubalis, n'a rien qui l'éloigne de sweetae.

Pour admettre la distinction entre $O$. cebei, parasite du même hôte à Hanoï, et $O$. sweetae, il faudrait démontrer que le diagnostic reposant sur la présence de papilles nettement précloacales a de la valeur.

\section{Bibliographie}

Bain O., Beveridge I. : Redescription d'Onchocerca gibsoni Cleland et Johnston, 1910. Ann. Parasitol. Hum. Comp., 1979, 54, 69-80.

Galliard H.: Onchocerca cebei, espèce nouvelle, parasite des Buffles du Tonkin. Ann. Parasitol. Hum. Comp., 1937, 15, 431-433.

Johnston T.H.: Onchocerciasis of Queensland cattle. Trans. Roy. Soc. South Austr., 1921, 45, 231-247.

Spratt D. M., Moorhouse D. E.: Onchocerca sweetae sp. nov. (Nematoda: Filarioidea), a parasite of the water buffalo (Bubalus bubalis) from Northern Australia. Zool. Anz., Leipzig, 1971, $186,147-153$.

Sweet G. : Investigation into the occurrence of Onchocerciasis in cattle and associated animals in countries other than Australia. Proc. Roy. Soc. Victoria, 1915, 28, 1-51.

* Il est difficile de prendre en considération la longueur des spicules, car sur les 2 spécimens mesurables, Sweet indique une longueur de $266 \mu \mathrm{m}$, donc intermédiaire entre les grands gibsoni et les petits sweetae. 